\title{
INELASTIC HYPERSPECTRAL LIDAR FOR AQUATIC ECOSYSTEMS MONITORING AND LANDSCAPE PLANT SCANNING TEST
}

\author{
Guangyu Zhao ${ }^{1,}$, Elin Malmqvist ${ }^{2}$, Klas Rydhmer ${ }^{2}$, Alfred Strand ${ }^{2}$, Giuseppe Bianco ${ }^{3}$, \\ Lars-Anders Hansson ${ }^{3}$ Sune Svanberg ${ }^{1,4}$, Mikkel Brydegaard, ${ }^{2,3}$ \\ ${ }^{1}$ Center of Optical and Electromagnetic Research, South China Normal University, Guangzhou, China \\ ${ }^{2}$ Combustion Physics Division, Department of Physics, Lund University, Sweden \\ ${ }^{3}$ Centre for Animal Movement, Department of Biology, Lund University, Sweden \\ ${ }^{4}$ Atomic Physics Division, Department of Physics, Lund University, Sweden \\ ${ }^{5}$ Norsk Elektro Optikk A/S, Norway \\ *guangyu.zhao@coer-scnu.org
}

\begin{abstract}
We have developed an aquatic inelastic hyperspectral lidar with unrestricted focal-depth and enough sensitivity and spatio-temporal resolution to detect and resolve position and behavior of individual sub-millimeter aquatic organisms. We demonstrate ranging with monitoring of elastic echoes, water Raman signals and fluorescence from chlorophyllbearing phytoplankton and dye tagged organisms. The system is based on a blue CW diode laser and a Scheimpflug optical arrangement.
\end{abstract}

\section{BACKGROUND}

Underwater suspended particle monitoring has an important role in aquatic ecosystem research. For instance, a specialized zooplankton Daphnia is a crucial and dominant species of the Arctic zone, and can serve as an indicator of conditions in polar area environments, which are particularly influenced by global change. There is considerable diversity in the layered vertical structures of salinity, illumination, and temperature as well as biomass distribution which exhibits rapid changes, e.g., the diel vertical migration of zooplankton shows changes even on a scale of one hour [1]. When it comes to monitoring these phenomena, remote sensing and real-time methods provide advantages over conventional methods, such as water sampling, which is laborious and intrusive.

Individual submillimeter organism detection is difficult to accomplish by sonar which is the most common and mature technique used in aquatic monitoring [2]. Laser optical particle counters (LOPC) and in-line digital holography are capable of detecting underwater sub- millimeter organisms with high precision $[3,4]$. However,

the monitoring area of these methods is limited, and such equipment is commonly installed on underwater vehicles to accomplish large area measurements. Light detection and ranging (lidar) techniques are readily used for atmospheric fauna and particle detection, as well as for aquatic monitoring with airborne systems. Recently, our group has developed atmospheric fauna lidar systems [5], which are based on high- power continuous-wave $(\mathrm{CW})$ laser diodes rather than pulsed lasers which are used in timeof-flight (TOF) lidar systems. TOF lidar has been widely used in atmospheric and topography monitoring with kilometer distance detection. However, rapidly varying particle monitoring is limited by the pulse width and repetition rate of the pulsed lasers used in TOF lidars [6]. In addition, lidar techniques are little developed for detailed feature assessment, e.g. of vertical structure, sub-millimeter organism size distribution, and fluorescence features. We here introduce a novel lidar technique, which provides hyperspectral monitoring and spatiotemporal resolution with a reasonable sensitivity [7].

\section{SYSTEM DESCRIPTION}

The system is based on a blue $\mathrm{CW}$ diode laser and a special optical arrangement. Range resolution can be achieved by using a Scheimpflug arrangement and employing the Hinge rule rather than the familiar time-of-flight approach. Based on the Scheimpflug lidar technique, hyperspectral detection is combined in the system to achieve range and spectral resolution simultaneously. The system provides 
enough sensitivity for underwater Raman as well as algae detection. The system shows advantages in cost, size and weight. A $1 \mathrm{~W}$ laser diode at $445 \mathrm{~nm}$ ( $3 \mathrm{~W}$ is commercially available) with a $1 \times 20 \mu \mathrm{m}$ chip element size was used in the current system. $445 \mathrm{~nm}$ is efficient to induce fluorescence of chlorophyll of phytoplankton (algae), and has potential to also detect constituents of zooplankton. A standard video objective ( $\varnothing 12 \mathrm{~mm}, \mathrm{f}_{\mathrm{exp}}=20 \mathrm{~mm}$ ) with adjustable focus and iris is used to collimate the laser light to focus the laser beam on the beam termination. The fast axis of the laser-diode chip is aligned in parallel with the baseline of the instrument as is indicated in Fig. 1. The smaller beam width (fast axis) is chosen to achieve a smaller beam width at the far range to compensate for the degraded range resolution. The whole transmission part is mounted with two rotational stages to adjust the orientation of the laser beam (shown in Fig. 1). To be able to achieve online background subtraction, the laser is modulated on and off, as triggered by a strobe signal from the detector,

Backscattered light is collected by an achromatic duplet lens (L1) and passes a $100 \mu \mathrm{m}$ slit. The slit is tilted by $45^{\circ}$. Then the light is collimated by a lens (L2) after the slit. A prism-gratingprism (PGP) optical assembly will disperse the light and image the light distribution on the sagittal plane of the imaging sensor. A lens (L3) is mounted after the PGP to focus the image on the detector. Correspondingly, the range information will be distributed in the meridian plane of the imaging sensor. Therefore, a 2D array (CCD) is used, which is a 12-bit industrial GigE camera with an active size of $7 \times 5 \mathrm{~mm}$. An appropriate long-pass filter is used to suppress the short wavelengths down to $1 \%$ to balance the relative intensities of the elastic and inelastic light. In the current system, the long-pass filter is inserted in the PGP component. The schematic of the system is shown in Fig. 1.

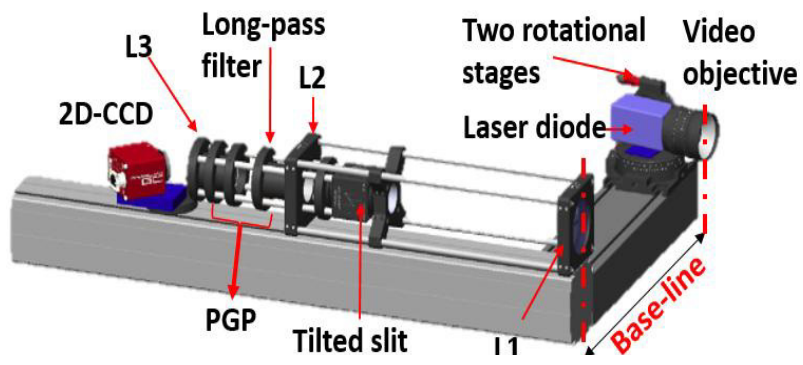

Fig. 1. Schematic of the inelastic hyperspectral lidar with its components.

The PGP is able to disperse the light in the spectral range 430-700 $\mathrm{nm}$ onto the CCD in the current system. The current system has 61 spectral bands, achieved even with a relatively wide slit, which allows detailed analysis of the spectra, e.g. Raman backscatter from liquid water. The wide spectral range gives the opportunity also for chlorophyll fluorescence monitoring. An experiment to demonstrate detection of phytoplankton, and labeled and unmarked zooplankton was performed. The advantage in low cost and small size could allow the system to be widely implemented for different layers of the ocean, as well as for shallow waters and polar environments. The system provides a novel non-intrusive tool for detecting aquatic plankton and suspended particulate matters.

\section{EXPERIMENT}

In order to evaluate the performance of the novel instrument, a tank which is $5 \mathrm{~m}$ long and supplied with transparent walls was used to provide a horizontal aquatic shooting range. The experimental arrangement is presented in Fig. 2.

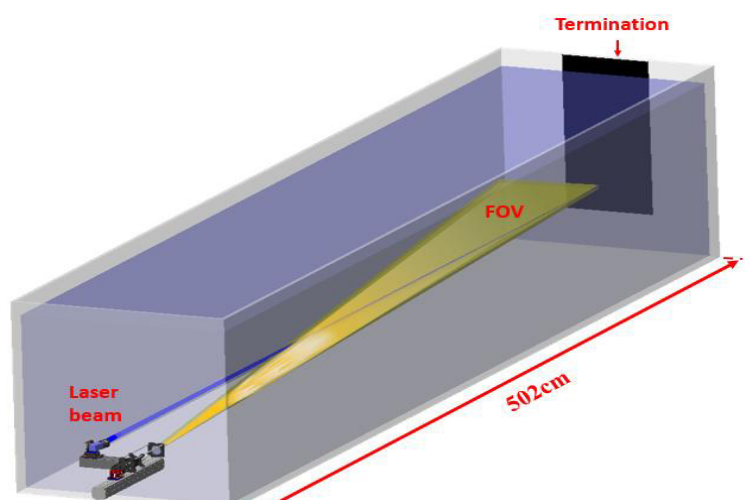

Fig. 2. The $5 \mathrm{~m}$ tank with the overlap between the laser beam and the FOV (field of view) indicated.

The tank was filled with clean freshwater. A black antistatic foam was used to terminate the laser beam with a resulting low intensity of the termination echo. The beam is focused on the termination from the $12 \mathrm{~mm}$ video objective aperture to a diffraction limited image of the laser chip. The volumetric backscatter signal of clean water and the $\mathrm{H}_{2} \mathrm{O}$ Raman signal are useful to help adjusting the best overlap between beam and FOV. The termination echo is used to 
calibrate the range [8]. A second-order polynomial fit on 3 laser lines $(445,532$, and $660 \mathrm{~nm}$ ) has been used to calibrate the spectral distribution, and this calibration was doublechecked by using the water O-H stretch Raman signal at $524 \mathrm{~nm}$, corresponding to a Raman shift of about $3400 \mathrm{~cm}^{-1}$ from the $445 \mathrm{~nm}$ laser line. A white halogen tungsten lamp, along with an integrated sphere, was used to calibrate the spectral response.

\section{RESULTS}

A 3D visualization of a seven-second recording is given in Fig. 3 which demonstrates the capability of the instrument.

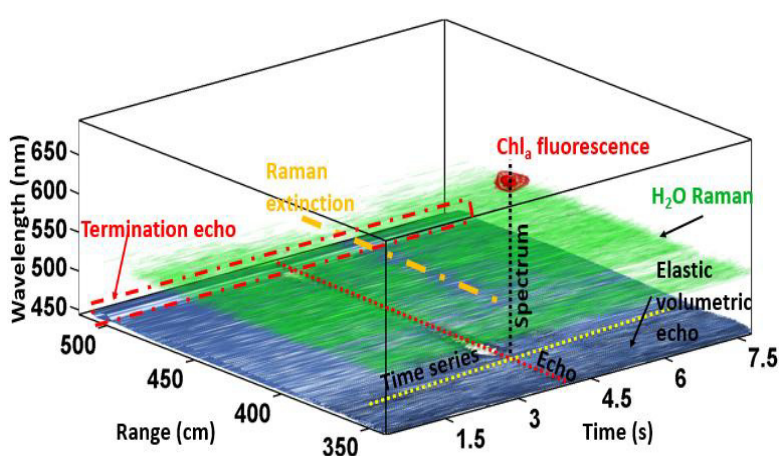

Fig. 3. 3D visualization of a 7 seconds data stream.

In Fig. 3, a drop of water containing phytoplankton was released close to the laser beam and the recording, which is represented by a data cube with an isosurface chosen with a strategic isolevel. The elastic, Raman and fluorescence signals are represented simultaneously. The volumetric elastic echo is shown as a blue layer, and the termination echo is observed and indicated in the figure. A Raman echo and fluorescence echo are shown by a green and a red layer, separately. Correspondingly, the termination echo could not be seen in the Raman and fluorescence bands. The red fluorescence of chlorophyll could be observed (red layer) when injecting the drop of water containing phytoplankton. In addition, corresponding elastic signals and the associated optical extinction (white hole in blue layer) are presented. The $\mathrm{H}_{2} \mathrm{O}$ Raman signal also shows a decrease due to the optical extinction of the phytoplankton from the injecting position to the termination. The spectral signals were recorded simultaneously, and a continuous monitoring could be achieved.
Fig. 5 shows the elastic, Raman and chlorophyll fluorescence curves of the static clean water and for water containing phytoplankton. The increase of the elastic echo and fluorescence signal due to

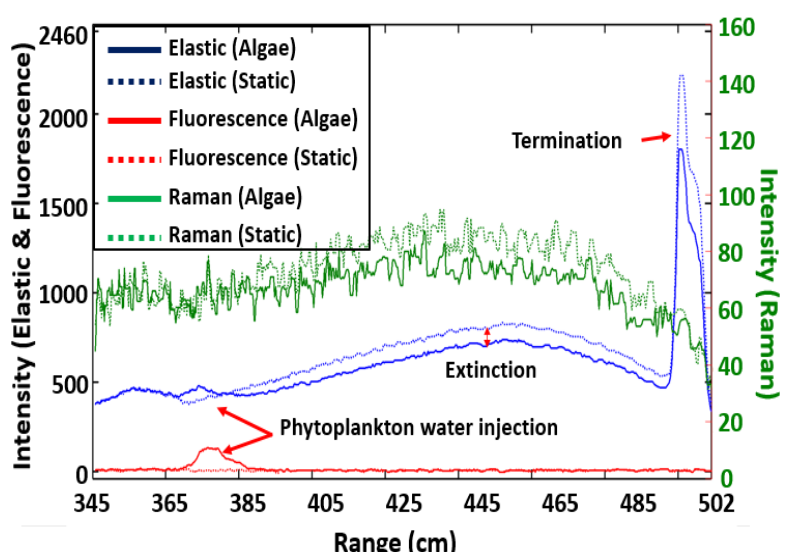

Fig. 4 Curves of elastic (445 nm), $\mathrm{H}_{2} \mathrm{O}$ Raman (524 $\mathrm{nm})$ and chlorophyll fluorescence $(680 \mathrm{~nm})$. Water containing phytoplankton was injected $370 \mathrm{~cm}$ from the entrance of the beam into the tank.

the algal release is noted. The optical extinction induced by the algae, which is noted above, is also represented. Consequently, the $\mathrm{H}_{2} \mathrm{O}$ Raman echo and the following decrease of the Raman signal are shown, from which it is possible to estimate the range resolved extinction coefficient.

In Fig. 5, unstained Daphnia and labeled Daphnia were injected into the lidar transect at distinct ranges and times. Fig. 5. C) shows the maximum, median and minimum echoes. These indicate rare zooplankton, static water volumetric echo and the noise level, respectively $[5,8,9]$. The time series of the three panels in Fig. 5. C) present the two unstained Daphnia and labeled Daphnia with yellow fluorescence.

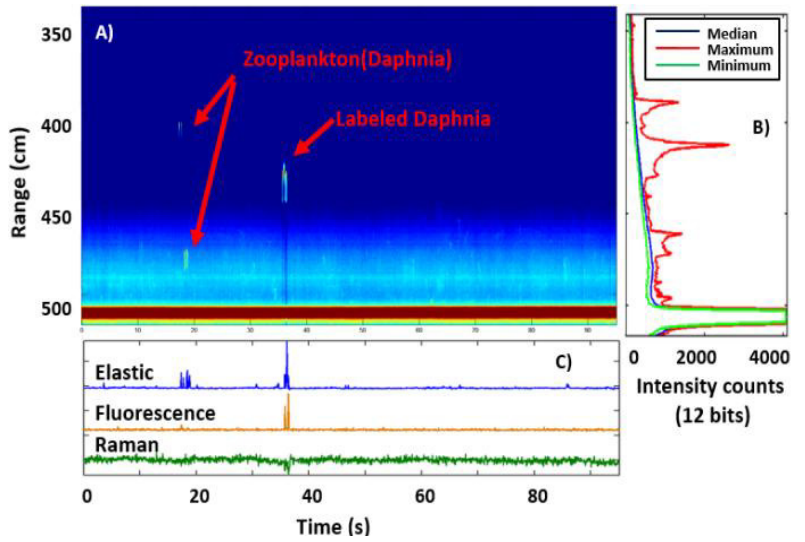


Fig. 5. A). Time-range map of the elastic echo of three Daphnia observations at different ranges and times. B) Minimum echo, median echo and maximum echo in a $100 \mathrm{~s}$ time window. C) Time-intensity curves of three spectral bands (445, 524 and 580 $n m)$.

\section{DISCUSSION, SUMMARY PERSPECTIVES}

AND

An inelastic hyperspectral lidar has been developed for an aquatic environment to detect and resolve the position and behavior of individual sub-millimeter aquatic organism. We demonstrate the performance of the instrument with monitoring of elastic echoes, water Raman signals and fluorescence from phytoplankton and dye labeled zooplankton with good range resolution. Hyperspectral detection is combined with continuous-wave lidar technique in the system to achieve range and spectral detection simultaneously. The system shows advantages in low cost, small size and light weight. The instrument provides a novel tool for nonintrusive monitoring in plankton research.

In addition, we are looking forward to using the device for canopy scanning in landscape. A coarse test of canopy monitoring has been performed on a small vegetation branch, and an example of the result is shown in Fig. 6. The elastic and chlorophyll fluorescence signals are displayed in different colors. 3-D shape and spectral information are achieved.

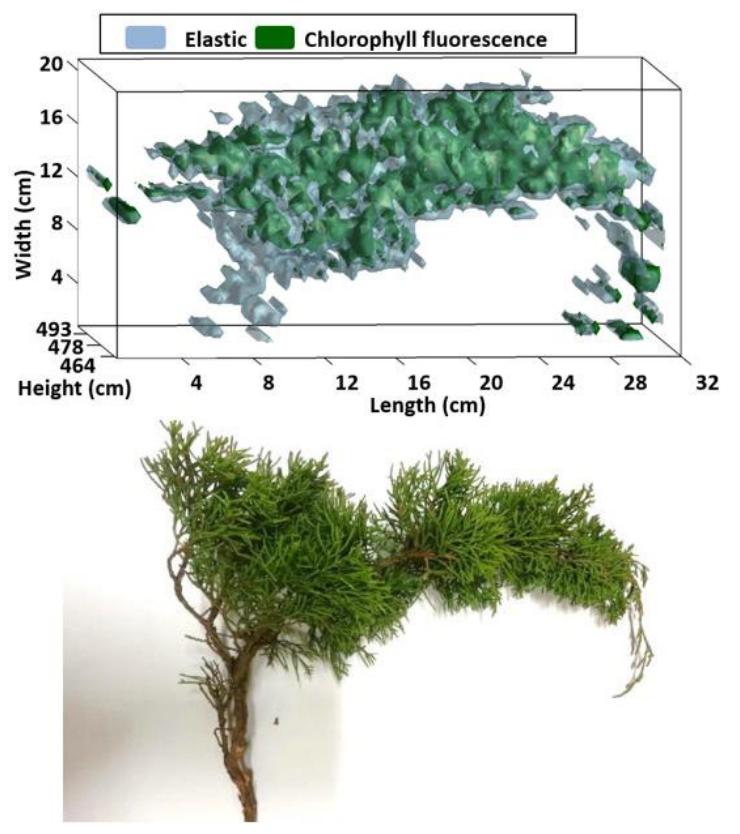

Fig. 6 3-D visualization of a scanned branch of Thuja.

\section{ACKNOWLEDVHGEMENTS}

The equipment could be acquired by an award from Edmund Optics. The work is supported by Lund University and the Overseas Study Program of Guangzhou Elite Project, and the Guangdong Province Innovation Research Team Program (No. 201001D0104799318).

\section{REFERENCES}

[1]. Lampert, W. Ultimate causes of diel vertical migration of zooplankton: new evidence for the predator-avoidance hypothesis. Diel Vertical Migration of Zooplankton, 39, 79$88,1993$.

[2]. Lemon, D., Johnston, P., Buermans, J., Loos, E., Borstad, G., \& Brown, L. (2012, October). Multiplefrequency moored sonar for continuous observations of zooplankton and fish. In Oceans, (pp. 1-6). IEEE, 2012.

[3]. Herman A W, Beanlands B, Phillips E F. The next generation of optical plankton counter: the laser-OPC. Journal of Plankton Research, 26(10): 1135-114, 2004.

[4]. Jericho, S. K., Garcia-Sucerquia, J., Xu, W. B., Jericho, M. H. \& Kreuzer, H. J. Submersible digital in-line holographic microscope. Rev. Sci. Instr. 77, doi:10.1063/1.2193827, 2006.

[5]. Brydegaard, M. Towards quantitative optical cross sections in entomological laser radar - Potential of temporal and spherical parameterizations for identifying atmospheric fauna. PLoS One 10, e0135231, 2015.

[6]. Wright, C. W., Hoge, F. E., Swift, R. N., Yungel, J. K. \& Schirtzinger, C. R. Next-generation NASA airborne oceanographic lidar system. Applied Optics 40, 336-342, 2001.

[7]. Zhao, G., Ljungholm, M., Malmqvist, E., Bianco, G., Hansson, L. A., Svanberg, S., \& Brydegaard, M. Inelastic hyperspectral lidar for profiling aquatic ecosystems. Laser \& Photonics Reviews, 10(5), 807-813, 2016.

[8]. Mei, L. \& Brydegaard, M. Continuous-wave differential absorption lidar. Laser \& Photonics Reviews 9, 629-636, doi:10.1002/lpor.201400419, 2015.

[9]. Brydegaard, M., Gebru, A., Kirkeby, C., Åkesson, S. \& Smith, H. Daily evolution of the insect biomass spectrum in an agricultural landscape accessed with lidar, in ILRC 27. New York, USA, 2015. 